\title{
Biomonitoring of selected trace elements in women, men and children from Slovenia
}

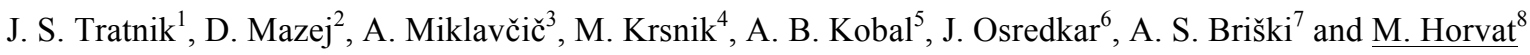 \\ ${ }^{1}$ Jožef Stefan Institute, Department of Environmental Sciences, Jamova cesta 39, Ljubljana, Slovenia, \\ janja.tratnik@ijs.si \\ ${ }^{2}$ Jožef Stefan Institute, Department of Environmental Sciences, Jamova cesta 39, Ljubljana, Slovenia, \\ darja.mazej@ijs.si \\ ${ }^{3}$ Jožef Stefan Institute, Department of Environmental Sciences, Jamova cesta 39, Ljubljana, Slovenia, \\ ana.miklavcic@ijs.si \\ ${ }^{4}$ University Medical Centre Ljubljana, Institute for clinical chemistry and biochemistry, Ljubljana, Slovenia, \\ mladen.krsnik@kclj-uni-lj.s \\ ${ }^{5}$ University Medical Centre Ljubljana, Institute for clinical chemistry and biochemistry, Ljubljana, Slovenia \\ ${ }^{6}$ University Medical Centre Ljubljana, Institute for clinical chemistry and biochemistry, Ljubljana, Slovenia, \\ josko.osredkar@kclj-uni-lj.s \\ ${ }^{7}$ University Medical Centre Ljubljana, Institute for clinical chemistry and biochemistry, Ljubljana, Slovenia, \\ alenka.briski@kclj-uni-lj.s \\ ${ }^{8}$ Jožef Stefan Institute, Department of Environmental Sciences, Jamova cesta 39, Ljubljana, Slovenia, \\ milena.horvat@ijs.si
}

\begin{abstract}
Little information is available on exposure of general population to metals at low levels. As a part of a research project PHIME within the EU $6^{\text {th }}$ Framework programme and National Human Biomonitoring programme, several studies following the same protocol were conducted to find out to what extent the Slovenian population of women in childbearing age, women 50-60 years, men and children 6-11 years is exposed to environmental chemicals including $\mathrm{Hg}, \mathrm{Pb}, \mathrm{Cd}$ and $\mathrm{As}$ and on the other hand to estimate their supply with essential elements ( $\mathrm{Se}, \mathrm{Zn}, \mathrm{Cu}$ ). Hair, blood and urine were collected from all participants, breast milk from lactating mothers. Most of the subjects had the levels for non-essential toxic metals below the reference levels and the levels for essential elements $\mathrm{Zn}, \mathrm{Cu}$ and $\mathrm{Se}$ within the reference intervals. Concentrations of metals and As did not differ between genders, while significantly higher levels of Se were observed in men and significantly higher levels of $\mathrm{Cu}$ in women. Comparing women of different ages, higher $\mathrm{Hg}$ levels and lower $\mathrm{Cd}$ and $\mathrm{Pb}$ levels were found in blood of younger women (20-35) than in older women (50-60). Se levels were higher in older women, while $\mathrm{Cu}$ and $\mathrm{Zn}$ levels were higher in younger women. In compare to children, women (both, younger and older) and men showed higher $\mathrm{Cd}, \mathrm{Pb}, \mathrm{Se}$ and $\mathrm{Zn}$ concentration in blood. Hg concentration was higher in women (20-35) and men than in children. Looking at the difference between different geographical areas where subjects were recruited, we found higher $\mathrm{Cd}$ and $\mathrm{Pb}$ levels in blood of rural children than in children from urban area. $\mathrm{Hg}$ in blood and urine was in contrary, higher in children from urban area than in children from rural area. In adults, As was observed to be the highest in urban area. Levels of essential elements differed between different areas in Slovenia as well. The present study has provided the basis to establish preliminary reference values for the selected population, depending on different parameters assessed by questionnaires. When additional subjects from other areas of Slovenia are recruited, the connection to the environmental database will be established using GIS modelling tools.
\end{abstract}

Key words: toxic elements, essential elements, human biomonitoring, Slovenia

\section{Introduction}

Little information is available on exposure of general population to metals at low levels. As a part of a research 
project Public health impact of long-term low-level mixed element exposure in susceptible population strata (PHIME) within the EU $6^{\text {th }}$ Framework programme and National Human Biomonitoring programme in Slovenia, several studies following the same protocol were conducted to find out to what extent the Slovenian population of women, men and children is loaded with environmental chemicals including mercury- $\mathrm{Hg}$, lead- $\mathrm{Pb}$, cadmium-Cd and arsenic-As and on the other hand to estimate their supply with essential elements (selenium-Se, zinc-Zn, and copper-Cu). Women were chosen because they are a sensitive group as they are often low in iron status which can affect absorption of other metals. Children were chosen as a sensitive group as well, while men where included as partners of women in childbearing age, as in general there is a lack of data on environmental chemical exposure in men. As the selected elements have not been determined in Slovenian population before, the present study has provided the basis to establish preliminary reference values for this population.

\section{Materials and Methods}

Women of childbearing age (20-35) and their partners were recruited from three geographically different areas in Slovenia: urban (the capital, Ljubljana), rural (Kočevje) and contaminated (Bela krajina, industrial activities in the past). Children aged 6-11 were recruited from urban (the capital, Ljubljana), rural and industrial area as well. Industrial area chosen in this case represented former mercury mine area (Idrija). Women aged 50-60 were recruited only in urban area, in the capital Ljubljana. Approximately 50 women $20-35,50$ men and 50 children were recruited from each of the selected area and 50 women 50-60 from the capital. In total 127 women 20-35, 147 men, 174 children and 66 women 50-60 were sampled for hair, blood, and urine, 117 lactating mothers collected breast milk samples as well. All participants were administered a short questionnaire on life style, work, medical status and food consumption. $\mathrm{Hg}$ was determined in hair, blood, urine and milk samples; $\mathrm{Cd}, \mathrm{Pb}$, $\mathrm{As}, \mathrm{Se}, \mathrm{Cu}$ and $\mathrm{Zn}$ were determined in blood and milk samples. The study was approved by the Commission for Medical Ethics of Republic of Slovenia.

$\mathrm{Hg}$ in hair and blood was determined by the Direct Mercury Analyser (DMA-80, Milestone Italy) integrating sample thermal combustion, amalgamation and detection by atomic absorption spectrometry. $\mathrm{Hg}$ in urine and milk was determined by semi-automated cold-vapour atomic absorption spectrometry. Other elements were determined by Inductively Coupled Plasma Mass Spectrometry (ICP-MS). Results in urine were corrected for creatinine levels - creatinine was determined by Jaffé reaction on biochemical analyser Dimension (Siemens). Markers of kidney damage (albumine, alpha-1-microglobuline, immunoglobuline $\mathrm{IgG}$ ) were determined as well, to control for co-excretion of metals in urine. Nephelometric immuno-chemical method (Siemens) was used.

\section{Results and Discussion}

Most of the subjects had the levels for non-essential toxic metals below the reference levels and the levels for essential elements $\mathrm{Zn}, \mathrm{Cu}$ and $\mathrm{Se}$ within the reference intervals. Levels of toxic metals and As determined in the selected population were lower than in other populations for which the data is published, and comparable to the general European population. Results are summarised in Table 1 .

Concentrations of metals and As did not differ between genders, while significantly higher levels of Se were observed in men and significantly higher levels of $\mathrm{Cu}$ in women $(\mathrm{p}<0.001)$. Comparing women of different ages, higher $\mathrm{Hg}$ levels and lower $\mathrm{Cd}$ and $\mathrm{Pb}$ levels were found in blood of younger women (20-35) than in women $50-60(p<0.001)$. Se levels were higher in older women $(\mathrm{p}=0.004)$, while $\mathrm{Cu}$ and $\mathrm{Zn}$ levels were higher in younger women $(\mathrm{p}<0.001)$. In compare to children, women (both, younger and older) and men showed higher $\mathrm{Cd}, \mathrm{Pb}, \mathrm{Se}$ and $\mathrm{Zn}$ concentration in blood $(\mathrm{p}<0.05)$. $\mathrm{Hg}$ concentration was significantly higher in women $(20-35)$ and men than in children $(\mathrm{p}<0.01)$. Looking at the difference between different geographical areas where subjects were recruited, we found higher $\mathrm{Cd}$ and $\mathrm{Pb}$ levels in blood of rural children than in urban children. $\mathrm{Hg}$ in blood and urine was in contrary, higher in urban children than in rural $(\mathrm{p}<0.05)$. In adults, As in blood, and also in breast milk, was observed to be the highest in urban area. Otherwise, levels of toxic elements did not differ between different areas. Levels of essential elements both in blood and in milk samples differed between different area. Based on the questionnaire data, $\mathrm{Cd}$ in children's blood was influenced the most by the density of traffic in vicinity of children's homes, while in women and men by smoking $(p<0.001)$. Regardless of area, $\mathrm{Hg}$ in blood and hair was influenced the most by frequency of fish consumption $(p<0.001$ and $p<0.05)$, while $\mathrm{Hg}$ in urine by number of amalgam fillings $(p<0.05)$. Se was statistically positively correlated with $\mathrm{Hg}$ in blood $(\mathrm{p}<0.001)$ in children from mercury mine area and in women and men. Traffic density or bone fractures in older women did not influence $\mathrm{Pb}$ concentration in blood $(p>0.05)$, but we found weak non-significant correlation between type of water supply and $\mathrm{Pb}$. Se was statistically positively correlated with $\mathrm{Hg}$ in blood $(p<0.001)$ in children from mercury mine area but not from other areas. Positive correlation was found also in women and men, whereat in men the correlation was stronger $\left(\mathrm{r}_{\mathrm{s}}=0.505, \mathrm{p}<0.001\right)$ than in women $\left(r_{s}=0.256, p<0.001\right)$. Zn was found not to be associated with either of the investigated elements.

\section{Conclusion}

The present study has provided the basis to establish preliminary reference values for this population, depending on different parameters assessed by 
Table. 1. Geometrical means for concentrations of toxic and essential elements in children, women and men from Slovenia.

\begin{tabular}{|c|c|c|c|c|}
\hline Analyte & Children, 6-11years & $\begin{array}{c}\text { Women, } \\
\mathbf{2 0 - 3 5} \text { years }\end{array}$ & $\begin{array}{c}\text { Women, } \\
\mathbf{5 0 - 6 0} \text { years }\end{array}$ & $\begin{array}{c}\text { Men, } \\
\mathbf{2 0 - 3 5} \text { years }\end{array}$ \\
\hline Hg in blood $(\mathrm{ng} / \mathrm{mL})$ & 0.77 & 1.04 & 0.76 & 1.11 \\
\hline Hg in hair $(\mathrm{ng} / \mathrm{g})$ & 183 & 241 & 324 & 216 \\
\hline Hg in urine $(\mu \mathrm{g} / \mathrm{g}$ creat.) & 0.73 & 0.73 & 0.62 & 0.37 \\
\hline Cd in blood $(\mathrm{ng} / \mathrm{mL})$ & 0.24 & 0.31 & 0.50 & 0.25 \\
\hline Pb in blood $(\mathrm{ng} / \mathrm{mL})$ & 16.1 & 17.3 & 26.7 & 19.6 \\
\hline As in blood $(\mathrm{ng} / \mathrm{mL})$ & 0.67 & 0.75 & 0.90 & 0.73 \\
\hline Se in blood $(\mathrm{ng} / \mathrm{mL})$ & 60 & 99 & 876 & 121 \\
\hline Cu in blood $(\mathrm{ng} / \mathrm{mL})$ & 845 & 998 & 5848 & 801 \\
\hline $\mathrm{Zn}$ in blood $(\mathrm{ng} / \mathrm{mL})$ & 4400 & 6791 & & 6618 \\
\hline
\end{tabular}

questionnaires. What do the observed concentrations for selected contaminants and essential elements actually mean in relation to human health will be discussed later on, when additional subjects from other areas of Slovenia are recruited (national human biomonitoring programme 2011-2014) and when the connection to the environmental database is established using GIS modelling tools.

\section{Acknowledgements}

This work was supported by the EU through its Sixth Framework Programme for RTD (contract no. FOOD-CT-2006-016253) and the National Human Biomonitoring programme in Slovenia (contract no. C2715-07Y000042) funded by Ministry of Health of Republic of Slovenia. 